(RESEARCH ARTICLE)

\title{
Distribution of Rothia species in root canals in a Japanese population
}

\author{
Teramoto Satoshi, Maeda Hiroshi *, Tsuji Norimasa, Ikenaga Hideaki and Yoshikawa Masataka \\ Department of Endodontics, School of Dentistry, Osaka Dental University 8-1 Kuzuhahanazonocho Hirakata, Osaka 573- \\ 1121, Japan.
}

Publication history: Received on 26 November 2019; revised on 06 December 2019; accepted on 09 December 2019

Article DOI: https://doi.org/10.30574/wjarr.2019.4.2.0095

\begin{abstract}
Rothia species are components of the normal oral microflora, which have been recognized as emerging opportunistic pathogens in immune compromised hosts. This study was performed to investigate the distribution of Rothia species in infected root canals. The correlations of clinical findings with the presence of Rothia species were also evaluated. A total of 200 root canal samples, including 92 primary infections and 108 secondary infections, were collected from 200 Japanese patients. Polymerase chain reaction (PCR) targeting the 16S rRNA gene was performed to detect three Rothia species, i.e., Rothia mucilaginosa, Rothia aeria, and Rothia dentocariosa. The detection frequencies of $R$. mucilaginosa, $R$. aeria, and $R$. dentocariosa were 49/200 (24.5\%), 95/200 (47.5\%), and 55/200 (27.5\%), respectively. There were no significant differences in the detection frequencies between primary and secondary endodontic infections. The detection frequency of $R$. mucilaginosa in cases with gingival swelling was $42.9 \%$, and there were significantly more cases positive than negative for this species $(\mathrm{P}=0.042)$. The presence of $R$. aeria also showed correlations with radiolucency around the root apex $(\mathrm{P}=0.001)$ and pain on percussion $(\mathrm{P}=0.044)$. Quantitative real-time PCR revealed that the relative abundance of $R$. mucilaginosa in total bacterial counts ranged from $0.04 \%$ to $91.8 \%$. These results indicated that Rothia species colonize root canals at relatively high frequency, and that root canals are potential reservoirs of these pathogens. The results also suggest that Rothia species are involved in the pathogenesis of apical periodontitis.
\end{abstract}

Keywords: Opportunistic pathogen; Endodontic infection; Rothia mucilaginosa; Rothia aeria; Rothia dentocariosa

\section{Introduction}

Rothia species are gram-positive cocobacilli present in the normal microflora of the oral environment and the upper respiratory tract. Although Rothia species (i.e., Rothia mucilaginosa, Rothia aeria, and Rothia dentocariosa) were originally believed to be of low virulence, these microorganisms are now recognized as pathogens involved in various systemic infections, such as endocarditis [1, 2], meningitis [3], pneumonia [4], bacteremia [5], knee joint infection [6], and endophthalmitis [7]. R. mucilaginosa, previously known as Stomatococcus mucilaginosus, has been most often reported as an emerging opportunistic pathogen involved in serious systemic infections. Immune compromised hosts, such as patients with hematological malignancies undergoing chemotherapy or neutropenic patients, have been reported as targets of this microorganism $[5,8]$.

Although the systemic infection routes of Rothia species have not been determined, they are presumed to involve gut translocation, catheter-related infection, or mucositis [8]. In addition to these routes, it is necessary to be aware of oral infectious diseases. Periodontitis and apical periodontitis are major oral infectious diseases, and $R$. mucilaginosa has been reported to be a pathogen involved in persistent periapical periodontitis [9]. Clinical isolates from root canals exhibit a dense meshwork-like structure on the cell surface, which has an appearance similar to a number of biofilmforming species [9]. To our knowledge, there have been no other investigations of the roles of Rothia species in the pathogenesis of apical periodontitis. The detection frequency in root canals has not been reported.

\footnotetext{
${ }^{*}$ Corresponding author

E-mail address: maeda-h@cc.osaka-dent.ac.jp
} 
Periodontitis, an infectious and inflammatory gum disease, is well known to be linked to various systemic diseases, such as cardiovascular disease, diabetes, and rheumatoid arthritis [10]. Apical periodontitis is a disease caused by polymicrobial infection of oral bacteria in the tooth root canal. Similar linkage between systematic diseases and apical periodontitis has recently attracted attention [11, 12,13]. However, the endodontic focal infection theory and the concept of endodontic medicine are still controversial [14]. The theory had been demised once, the question whether the apically detected inflammation or bacteria is a threat to systemic health has again been raised [14].

Small apical lesions with small amounts of microorganisms may not affect the systemic condition in healthy individuals. However, in immunocompromised hosts, such as neutropenic patients, root canals connected to alveolar bone could act as a route of oral bacterial infection, and may have deleterious effects on morbidity and mortality. Indeed, endodontic pathogens have been reported to be causes of serious systemic infections $[15,16]$. This study was performed to investigate the detection frequency of Rothia species in infected root canals and the associations with the clinical symptom of apical periodontitis.

\section{Material and methods}

\subsection{Sample collection}

A total of 200 patients treated at Osaka Dental University Hospital for root canal treatment, who did not have any systemic diseases, and had not received antibiotic treatment during the past 3 months were selected for this study. The patients ranged in age from 20 to 93 years. One tooth was selected from each patient. Ninety-two teeth showed necrotic pulp tissue and 108 teeth had received root canal treatment but showed inadequate obturation with obvious dead space in X-ray findings. The presence of radiolucency at the apex, gingival swelling, spontaneous pain, sinus tract, and pain on percussion were examined on the sampling date. The Ethics Committee of Osaka Dental University approved the protocol of this study (approval number: 110972), and all patients signed an informed consent form to participate in this study.

Prior to sampling, the teeth were isolated with a rubber dam and were disinfected with $30 \% \mathrm{H}_{2} \mathrm{O}_{2}$ and $2.5 \% \mathrm{NaOCl}$ after removing dental caries. Followed by neutralization with $5 \%$ sodium thiosulfate, an access cavity was prepared using sterile diamond burs under irrigation with sterile saline. In the case of retreatment, restorations were removed and the disinfection protocol was repeated before removing the root canal obturation. Pre-existing root filling materials were removed using endodontic files without the use of gutta percha solvents. Bacterial samples were collected using endodontic files (K-files: Mani Inc.) with corresponding in size to the diameter of the root canals near the apex. After repeating the filling operation several times, the contents and the dentin powder of the root canals attached to the file were suspended in $1 \mathrm{ml}$ of phosphate-buffered saline (PBS) (Invitrogen). Aseptic technique was employed throughout the sampling procedure. The bacterial samples were stored at $4^{\circ} \mathrm{C}$ and were subjected to DNA extraction on the same day.

\subsection{DNA extraction}

InstaGene Matrix (Bio-Rad) was used for DNA extraction from the samples according to the manufacturer's instructions. Briefly, bacterial cells suspended in PBS were pelleted by centrifugation (14000 rpm for 10 minutes) and resuspended in $200 \mu \mathrm{l}$ of InstaGene Matrix. The suspensions were incubated at $56^{\circ} \mathrm{C}$ for 30 minutes and then $100^{\circ} \mathrm{C}$ for 8 minutes. After incubation, the suspensions were centrifuged and aliquots of $2 \mu$ of the resulting supernatants were used for PCR and real-time PCR.

\subsection{Detection of Rothia species (PCR method)}

Three Rothia species, i.e., $R$. mucilaginosa, $R$. aeria, and $R$. dentocariosa, were detected by PCR according to the method described by Tsuzukibashi et al. [17]. The PCR products were electrophoresed on 1.5\% agarose gels, stained with ethidium bromide $(50 \mu \mathrm{g} / \mathrm{ml})$, and the products were detected under UV illumination (302 $\mathrm{nm})$.

\subsection{Quantitative detection of $R$. mucilaginosa and total bacteria (real-time PCR method)}

Total bacterial counts in the samples were determined by real-time PCR as described previously [18]. For quantitative detection of $R$. mucilaginosa, we designed a primer set for real-time PCR. $R$. mucilaginosa-specific regions on the $16 \mathrm{~S}$ rDNA were identified by alignment of the published sequence data of $16 \mathrm{~S}$ rDNA of other bacterial species, including $R$. aeria and $R$. dentocariosa, obtained from the National Center for Biotechnology Information website and Human Oral Microbiome Database. Alignments were performed in Genetyx ver. 8 (Genetyx). Prior to application to the clinical specimens, quantitativity and specificity of the primers were confirmed as described previously [18] (data not shown). 
The nucleotide sequences of the primers were as follows: forward primer, $5^{\prime}$-GAAGAGARATTGACGGTACC-3' ; reverse primer, $5^{\prime}$-CCGTACCCACTGCAATACAC-3' . Real-time PCR was performed using PowerUp ${ }^{\mathrm{TM}}$ SYBR $^{\mathrm{TM}} \mathrm{Green}^{\prime}$ Master Mix (Thermo Fisher Scientific) and QuantStudio ${ }^{\mathrm{TM}} 3$ (Thermo Fisher Scientific). The reaction mixture contained $300 \mathrm{nM}$ of each primer and $2 \mu \mathrm{l}$ of sample DNA in a total volume of $20 \mu \mathrm{l}$. The thermal cycling profile was as follows: $50^{\circ} \mathrm{C}$ for 2 minutes (Uracil-DNA-glycosylase (UDG) activation step), $95^{\circ} \mathrm{C}$ for 2 minutes (activation step for Dual-lock ${ }^{\mathrm{TM}}$ DNA polymerase), and 35 cycles of $95^{\circ} \mathrm{C}$ for $10 \mathrm{~s}$ and $60^{\circ} \mathrm{C}$ for $30 \mathrm{~s}$. Melting curve analysis was performed in the range of $60^{\circ} \mathrm{C}$ to $95^{\circ} \mathrm{C}$ for each reaction to assess the specificity. $R$. mucilaginosa DY-18 was cultivated on blood agar plates (BBL Microbiology Systems). Bacterial colonies on the agar plates were suspended in PBS and subjected to DNA extraction. The DNA samples equivalent to $10-10^{6}$ bacterial cells were used to construct a standard curve.

\subsection{Statistical analysis}

The significance of differences in frequency of Rothia-positive subjects between the presence or absence of the individual clinical findings was assessed by Fisher's Exact Test. In all analyses, $P<0.05$ was taken to indicate statistical significance.

\section{Results}

\subsection{Detection frequency of the three Rothia species}

A total of 200 samples were obtained from the patients. Table 1 shows the distribution of the three Rothia species in primary and secondary endodontic infections. Total detection frequencies of $R$. mucilaginosa, $R$. aeria, and $R$. dentocariosa in infected root canals were $24.5 \%, 47.5 \%$, and $27.5 \%$, respectively. In $134 / 200$ cases (67\%), at least one of the Rothia species was detected in the root canals. There were no significant differences in detection frequency between primary and secondary endodontic infections.

Table 1 The prevalence of Rothia species in primary and secondary root canal infection

\begin{tabular}{lllll}
\hline & $\begin{array}{l}\boldsymbol{R} . \\
\text { mucilaginosa }\end{array}$ & R. aeria & R. dentocariosa & $\begin{array}{l}\text { Including at least one } \\
\text { Rothia species }\end{array}$ \\
\hline $\begin{array}{l}\text { primary } \\
\text { infection } \\
(\mathrm{n}=92)\end{array}$ & $24(26.1 \%)$ & $43(46.7 \%)$ & $24(26.1 \%)$ & $61(66.3 \%)$ \\
$\begin{array}{l}\text { secondary } \\
\text { infection } \\
(\mathrm{n}=108)\end{array}$ & $25(23.1 \%)$ & $52(48.1 \%)$ & $31(28.7 \%)$ & $74(68.5 \%)$ \\
Total (n = 200) & $49(24.5 \%)$ & $95(47.5 \%)$ & $55(27.5 \%)$ & $134(67.0 \%)$ \\
\hline
\end{tabular}

\subsection{Distribution of Rothia species and clinical findings}

The distribution of the three Rothia species in cases exhibiting radiolucency around the root apex, gingival swelling, pain on percussion, spontaneous pain, and sinus tract are shown in Table 2. The detection frequency of $R$. mucilaginosa in cases with gingival swelling was $42.9 \%$. There were significantly more cases of gingival swelling positive than negative for $R$. mucilaginosa $(P=0.042)$. There were significantly more cases exhibiting radiolucency at the apex positive than negative for $R$ aeria $(P=0.001)$. As with radiolucency, there was a significant correlation between the presence of $R$. aeria and pain on percussion $(P=0.044)$. Among the total of 200 subjects, 141 subjects exhibited at least one clinical finding, and there were significantly more of these cases positive than negative for $R$. aeria $(P=0.001)$. Fiftynine subjects exhibited no clinical findings. The detection frequencies of $R$. mucilaginosa, R. aeria, and $R$. dentocariosa in this group were $16.9 \%, 30.5 \%$, and $30.5 \%$, respectively (data not shown in the table). 
Table 2 Clinical findings and prevalence of Rothia species

\begin{tabular}{|c|c|c|c|c|c|c|c|}
\hline & $\begin{array}{l}\text { Total } \\
\text { sample } \\
(n=200)\end{array}$ & $\begin{array}{l}\text { Radio- } \\
\text { lucency } \\
(n=117)\end{array}$ & $\begin{array}{l}\text { Swelling } \\
(n=21)\end{array}$ & $\begin{array}{l}\text { Percussio } \\
\text { n pain } \\
(n=67)\end{array}$ & $\begin{array}{l}\text { Spontane } \\
\text { ous pain } \\
(n=19)\end{array}$ & $\begin{array}{l}\text { Sinus } \\
\text { tract } \\
(n=39)\end{array}$ & $\begin{array}{l}\text { Cases } \\
\text { including } \\
\text { at least } \\
\text { one } \\
\text { finding ( } n \\
=141 \text { ) }\end{array}$ \\
\hline $\begin{array}{l}R . \\
\text { mucilagin } \\
\text { osa }\end{array}$ & $\begin{array}{l}49 \\
(24.5 \%)\end{array}$ & $\begin{array}{l}33 \\
(28.2 \%)\end{array}$ & $\begin{array}{l}9 * \\
(42.9 \%)\end{array}$ & $\begin{array}{l}19 \\
(28.4 \%)\end{array}$ & $\begin{array}{l}5 \\
(26.3 \%)\end{array}$ & $\begin{array}{l}13 \\
(33.3 \%)\end{array}$ & $\begin{array}{l}39 \\
(27.7 \%)\end{array}$ \\
\hline $\begin{array}{l}R . \\
\text { aeria }\end{array}$ & $\begin{array}{l}95 \\
(47.5 \%)\end{array}$ & $\begin{array}{l}68 * \\
(58.1 \%)\end{array}$ & $\begin{array}{l}13 \\
(61.9 \%)\end{array}$ & $\begin{array}{l}38^{*} \\
(56.7 \%)\end{array}$ & $\begin{array}{l}9 \\
(47.4 \%)\end{array}$ & $\begin{array}{l}21 \\
(53.8 \%)\end{array}$ & $\begin{array}{l}77^{*} \\
(54.6 \%)\end{array}$ \\
\hline $\begin{array}{l}R . \\
\text { dentocario } \\
\text { sa }\end{array}$ & $\begin{array}{l}55 \\
(27.5 \%)\end{array}$ & $\begin{array}{l}32 \\
(27.4 \%)\end{array}$ & $\begin{array}{l}7 \\
(33.3 \%)\end{array}$ & $\begin{array}{l}19 \\
(28.4 \%)\end{array}$ & $\begin{array}{l}3 \\
(15.8 \%)\end{array}$ & $\begin{array}{l}7 \\
(17.9)\end{array}$ & $\begin{array}{l}37 \\
(26.2 \%)\end{array}$ \\
\hline
\end{tabular}

\subsection{Relative abundance of $R$. mucilaginosa in total bacterial counts}

The 49 samples positive for R. mucilaginosa were subjected to real-time PCR. Total bacterial counts and cell numbers of $R$. mucilaginosa were quantified, and the relative abundance of $R$. mucilaginosa in each sample was calculated. The ratio of $R$. mucilaginosa in total bacterial counts ranged from $0.04 \%$ to $91.8 \%$. Table 3 shows the distribution of $R$. mucilaginosa-positive subjects divided according to the range of the ratio. In 16 (32.7\%) root canals, the ratio of $R$. mucilaginosa was $<1 \%$, and the ratio was $1 \%-5 \%$ in $14(28.6 \%)$ root canals. The ratio of $R$. mucilaginosa in the total bacterial counts was $>20 \%$ in 10 root canals $(20.4 \%)$. There were no statistically significant correlations between relative abundance and clinical findings (data not shown).

Table 3 Relative abundance of $R$. mucilaginosa in total bacterial counts

\begin{tabular}{|c|c|c|c|c|c|c|}
\hline & \multirow[t]{2}{*}{$<1 \%$} & $1 \%-$ & $5 \%-$ & $10 \%-$ & $15 \%-$ & \multirow[t]{2}{*}{$>20 \%$} \\
\hline & & $\begin{array}{l}\text { less than } \\
5 \%\end{array}$ & $\begin{array}{l}\text { less than } \\
10 \%\end{array}$ & $\begin{array}{l}\text { less than } \\
15 \%\end{array}$ & $\begin{array}{l}\text { less than } \\
20 \%\end{array}$ & \\
\hline $\begin{array}{l}\text { Number of } \\
\text { subjects }\end{array}$ & $16(32.7)$ & $14(28.6)$ & $3(6.1)$ & $4(8.2)$ & $2(4.1)$ & $10(20.4)$ \\
\hline $\begin{array}{l}(\%), \text { total } n= \\
49\end{array}$ & & & & & & \\
\hline
\end{tabular}

\section{Discussion}

Serious systemic infection caused by Rothia species has become a significant clinical concern, especially in immune compromised hosts [5]. An increasing number of systemic infectious diseases caused by these microorganisms have been reported [1 - 7]. Rothia species are components of the normal oral microflora, and R. mucilaginosa has been suggested to be involved in the pathogenesis of apical periodontitis [9]. However, there have been no studies regarding endodontic infections as a reservoir of Rothia species. Although gut translocation, catheter-related infection, and mucositis are estimated to be the main infection routes [8], to our knowledge, there have been no detailed studies of this issue. Root canals connected to alveolar bone represent a potential reservoir of Rothia species. The main purpose of this study was to raise awareness regarding the potential risk of infected root canals as a reservoir of Rothia species by determining their distribution.

Among 200 root canals examined, 134 (67.0\%) contained at least one Rothia species. Among the three Rothia species examined, $R$. aeria was detected at the highest rate $(47.5 \%)$ in this study population. They were detected in both primary and secondary root canal infections at similar rates. Enterococcus faecalis is one of the pathogens most commonly implicated in endodontic infections, and a series of reports have investigated its distribution [19]. Dumani et al. [20] 
reported detection of $E$. faecalis in 19/117 primary infections and in 11/114 persistent infections by a PCR-based method. By real-time PCR, another study demonstrated the presence of E. faecalis in 27/40 primary infections and 43/48 persistent infections [21]. Although the detection frequencies of $E$. faecalis differed between the reports, in comparison to these previous studies using PCR-based methods, Rothia species were detected at relatively high frequency in root canals in the present study. They may be among the most common components of the microbial communities. Further studies using culture methods are needed to further elucidate their viability.

The correlations between the presence of Rothia species and clinical findings were examined in the present study. We have previously isolated $R$. mucilaginosa from persistent apical periodontitis lesions [9]. The isolated $R$. mucilaginosa exhibited a dense biofilm with a meshwork-like structure, suggesting the involvement of this microorganism in the pathogenesis of endodontic infection. The results of the present study suggest an association with gingival swelling. With regard to the associations with clinical findings, $R$. aeria is more likely to be correlated to clinical findings than $R$. mucilaginosa. The results suggested correlations of the presence of $R$. aeria with radiolucency and pain on percussion. It is still unknown whether Rothia species directly show virulence in apical periodontitis. Synergistic effects with other bacterial species in the microflora should also be examined in further studies. In addition, virulence factors of the Rothia species have not been determined, as the bacteria had been considered to have low virulence. It will be necessary to determine how the Rothia species are selected and to examine their virulence as opportunistic pathogens in immune compromised hosts.

Among the three Rothia species, $R$. mucilaginosa has been reported most frequently as a cause of systemic infections. Ramanan et al. [8] reported the frequency of $R$. mucilaginosa infection among neutropenic and leukemia patients. They reported that among 25 patients positive for Rothia in blood culture tests, $R$. mucilaginosa was responsible for all of the bloodstream infections. Therefore, we attempted to quantify the bacterial species in root canals. The detected counts of $R$. mucilaginosa ranged from 50 to $2.7 \times 10^{4}$. As it is not possible to collect the bacterial cells entirely from the root canals, the bacterial number itself is not important for the analysis, so the relative abundance of $R$. mucilaginosa in total bacterial counts was determined in this study. Among the 49 patients positive for $R$. mucilaginosa, the ratio was $<1 \%$ in 16 patients and $>20 \%$ in 10 patients. These results suggested that $R$. mucilaginosa is a major component of the root canal microflora in some cases.

In this study, systemically healthy subjects were selected, and the distribution of Rothia species in root canals of immune compromised hosts has yet to be elucidated. Favorable host factors for $R$. mucilaginosa, such as long-term neutropenia followed by chemotherapy and repeated or prolonged exposure to broad-spectrum antibiotics, have been suggested to lead to the selection of this pathogen $[8,22]$. Therefore, the detection frequency and the ratio of Rothia species in root canals or oral microflora of immune compromised hosts may be higher than in healthy subjects. Further research regarding the distribution of Rothia in immune compromised hosts is urgently required.

We determined the detection frequencies of the three Rothia species in infected root canals. In root canals, the Rothia species were detected at relatively high frequency. The results indicated that the root canals represent a potential infection route for these pathogens to alveolar bone and the whole body. The results presented here suggest that bacterial examination of root canals should be performed in immune compromised hosts, especially in cases of bacteremia. Indeed, dental diseases have been suggested to be a cause of Rothia infection [6], although the details are still unclear. Genotyping approaches will be required to answer these questions. Endodontic focal infection theory has once been denied. However, recent advances in endodontic therapy and bacterial examination technology have enabled us to eliminate the infection of root canals without tooth extraction. Dentists, especially endodontists, must identify the risk of root canal infection and determine the necessity of treatment, especially in cases of hematological malignancy prior to chemotherapy or hematopoietic stem cell transplantation.

\section{Conclusion}

The results of this study showed that three Rothia species, i.e., $R$. mucilaginosa, $R$. aeria, and $R$. dentocariosa, are distributed in root canals at relatively high rates. These opportunistic pathogens may be involved in the pathogenesis of apical periodontitis, and the root canals may represent a potential reservoir. 


\section{Compliance with ethical standards}

\section{Acknowledgments}

This study was supported in part by a Grant-in-Aid for Scientific Research (Grant number: 18K09613) from the Japan Society for the Promotion of Science.

\section{Disclosure of conflict of interest}

The authors have no conflicts of interest to declare.

\section{References}

[1] Bruminhent J, Tokarczyk MJ, Jungkind D and Desimone JA Jr. (2013). Rothia mucilaginosa prosthetic device infections: a case of prosthetic valve endocarditis. J Clin Microbiol, 51, 1629 - 1632.

[2] Willner S, Imam Z and Hader I. (2019). Rothia dentocariosa endocarditis in an unsuspecting host: A case report and literature review. Case Rep Cardiol, 7464251.

[3] Lee AB, Harker-Murray P, Ferrieri P, Schleiss MR and Tolar J. (2008). Bacterial meningitis from Rothia mucilaginosa in patients with malignancy or undergoing hematopoietic stem cell transplantation. Pediatr Blood Cancer, 50, 673 - 676 .

[4] Maraki S and Papadakis IS. (2015). Rothia mucilaginosa pneumonia: a literature review. Infect Dis (Lond), 47, 125-129.

[5] Abidi MZ, Ledeboer N, Banerjee A and Hari P. (2016). Morbidity and mortality attributable to Rothia bacteremia in neutropenic and nonneutropenic patients. Diagn Microbiol Infect Dis, 85, 116-120.

[6] Mahobia N, Chaudhary P and Kamat Y. (2013). Rothia prosthetic knee joint infection: report and mini-review. New Microbes New Infect, 1, 2-5.

[7] Oie S, Mochizuki K, Ishida K, Nakayama A and Ohkusu K. (2016). Case of late-onset bleb associated endophthalmitis caused by Rothia mucilaginosa. J Infect Chemother, 22, 645-647.

[8] Ramanan P, Barreto JN, Osmon DR and Tosh PK. (2014). Rothia bacteremia: a 10-year experience at Mayo Clinic, Rochester, Minnesota. J Clin Microbiol, 52, 3184-3189.

[9] Yamane K, Yoshida M, Fujihira T, Baba T, Tsuji N, Hayashi H, Sugimori C, Yamanaka T, Mashimo C, Nambu T, Kawai H and Fukushima H. (2010). Isolation and identification of Rothia mucilaginosa from persistent apical periodontitis lesions. J Osaka Dent Univ, 44(2), 93-98.

[10] Kumar PS. (2016). From focal sepsis to periodontal medicine: a century of exploring the role of the oral microbiome in systemic disease. J Physiol, 595, 465-476.

[11] Segura-Egea JJ, Martín-González J and Castellanos-Cosano L. (2015). Endodontic medicine: connections between apical periodontitis and systemic diseases. Int Endod J, 48(10), 933-51.

[12] Khalighinejad N, Aminoshariae MR, Aminoshariae A, Kulild JC, Mickel A and Fouad AF. (2016). Association between Systemic Diseases and Apical Periodontitis. J Endod, 42(10), 1427-1434.

[13] Virtanen E, Nurmi T, Söder P, Airila-Månsson S, Söder B and Meurman JH. (2017). Apical periodontitis associates with cardiovascular diseases: a cross-sectional study from Sweden. BMC Oral Health, 17, 107.

[14] Tjäderhane L. (2015). Endodontic infections and systemic health - where should we go? Int Endod J, 48, 911-912.

[15] Poeschl PW, Crepaz V, Russmueller G, Seemann R, Hirsch AM and Ewers R. (2011). Endodontic pathogens causing deep neck space infections: clinical impact of different sampling techniques and antibiotic susceptibility. J Endod, $37(9), 1201-1205$.

[16] Blount CA and Leser C. (2012). Multisystem complications following endodontic therapy. J Oral Maxillofac Surg, $70(3), 527-530$.

[17] Tsuzukibashi O, Uchibori S, Shinozaki-Kuwahara N, Saito M, Kobayashi T and Fukumoto M. (2013). New primer design for identification of oral rothia, including R. aeria, using multiplex PCR. Int J Oral-Med Sci, 12, 85-89. 
[18] Maeda H, Fujimoto C, Haruki Y, Maeda T, Kokeguchi S, Petelin M, Arai H, Tanimoto I, Nishimura F and Takashiba S. (2003). Quantitative real-time PCR using TaqMan and SYBR Green for Actinobacillus actinomycetemcomitans, Porphyromonas gingivalis, Prevotella intermedia, tetQ gene and total bacteria. FEMS Immunol Med Microbiol, 39, 81-86.

[19] Zhang C, Du J and Peng Z. (2015). Correlation between Enterococcus faecalis and persistent intraradicular infection compared with primary intraradicular infection: A Systematic Review. J Endo, 41(8), 1207-1213.

[20] Dumani A, Yoldas O, Yilmaz S, Koksal F, Kayar B, Akcimen B and Seydaoglu G. (2012). Polymerase chain reaction of Enterococcus faecalis and candida albicans in apical periodontitis from Turkish patients. J Clin Exp, 4(1), e3439.

[21] Sedgley C, Nagel A, Dahlén G, Reit C and Molander A. (2006). Real-time quantitative polymerase chain reaction and culture analyses of Enterococcus faecalis in root canals. J Endod, 32(3), 173-177.

[22] Clauwaert M, Druwé P and Depuydt P. (2019). Meningitis in a patient with neutropenia due to Rothia mucilaginosa: a case report. J Med Case Rep, 13(1), 84.

\section{How to cite this article}

Teramoto S, Maeda H, Tsuji N, Ikenaga H and Yoshikawa M. (2019). Distribution of Rothia species in root canals in a Japanese population. World Journal of Advanced Research and Reviews, 4(2), 20-26. 